\title{
Spin Motion and Resonances In Accelerators and Storage Rings
}

\author{
E.D. Courant
}

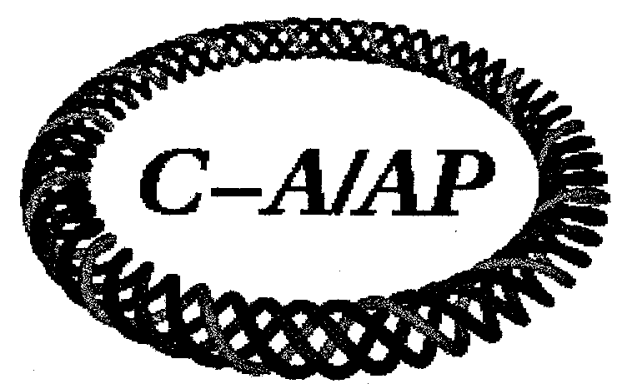

Collider-Accelerator Department Brookhaven National Laboratory Upton, NY 11973

Notice: This document has been authorized by employees of Brookhaven Science Associates, LLC under Contract No. DE-AC02-98CH10886 with the U.S. Department of Energy. The United States Government retains a nonexclusive, paid-up, irrevocable, world-wide license to publish or reproduce the published form of this document, or allow others to do so, for United States Government purposes. 


\title{
Spin Motion and Resonances in Accelerators and Storage Rings
}

\author{
E D Courant
}

\begin{abstract}
Some of the basic aspects of the spin dynamics of accelerators and storage rings are reviewed. Since the components of spin parallel and perpendicular to the particle velocity behave differently it is desirable to reformulate the equations of spin motion in a frame of reference that exhibits this difference explicitly. The conventional treatment employs a coordinate system derived from a reference orbit. An alternate coordinate system, based on the actual trajectory of the particle, leads to simplified equations of spin motion but, contrary to a conjecture presented in a previous note, resonance strengths calculated by the conventional and the revised formalisms are identical, as pointed out by Kondratenko. Resonances induced by radiofrequency dipoles or solenoids are also treated; with rf dipoles it is essential to consider not only the direct effect of the dipole but also the contribution from oscillations induced by it.
\end{abstract}




\section{Fundamentals}

Froissart and Stora's [1] formulation of the Thomas-BMT equation [2] for the behavior of spin in static magnetic fields may be written

$$
\frac{d \vec{S}}{d t}=\frac{q}{m \gamma} \vec{S} \times\left[\vec{B}+G\left(\gamma \vec{B}_{\perp}+\vec{B}_{\|}\right)\right]
$$

where $\vec{B}_{\|}=(\hat{\mathrm{v}} \cdot \vec{B}) \hat{\mathrm{v}}$ and $\vec{B}_{\perp}=\vec{B}-\vec{B}_{\|}=(\hat{\mathrm{v}} \times \vec{B}) \times \hat{\mathrm{v}}$ are the longitudinal and transverse parts of the magnetic field $\vec{B}, \hat{\mathrm{v}}$ being the unit vector in the direction of the particle velocity; $G \equiv(g-2) / 2$ is the magnetic moment anomaly of the particle, $q$ and $m$ are its charge and mass, and $\gamma$ is the Lorentz energy factor. Similarly the longitudinal and transverse parts of the spin are $\vec{S}_{\|}=(\hat{\mathrm{v}} \cdot \vec{S}) \hat{\mathrm{v}}$ and $\vec{S}_{\perp}=\vec{S}-\vec{S}_{\|}=(\hat{\mathrm{v}} \times \vec{S}) \times \hat{\mathrm{v}}$.

The Lorentz force equation gives

$$
\frac{d \hat{\mathrm{v}}}{d t}=\frac{q}{m \gamma} \hat{\mathrm{v}} \times \vec{B}
$$

Combining (1.1) and (1.2) we obtain

$$
\begin{aligned}
\frac{d \vec{S}}{d t} & =\frac{q}{m \gamma} \vec{S} \times\left[\vec{B}+G\left(\gamma \vec{B}_{\perp}+\vec{B}_{\|}\right)\right] \\
\vec{S}_{\|} & =(\hat{\mathrm{v}} \cdot \vec{S}) \hat{\mathrm{v}} \text { and } \vec{S}_{\perp}=\vec{S}-\vec{S}_{\|}=(\hat{\mathrm{v}} \times \vec{S}) \times \hat{\mathrm{v}} \\
\frac{d \vec{S}_{\|}}{d t} & =\left(\frac{d \vec{S}}{d t} \cdot \hat{\mathrm{v}}+\vec{S} \cdot \frac{d \hat{\mathrm{v}}}{d t}\right) \hat{\mathrm{v}}+(\hat{\mathrm{v}} \cdot \vec{S}) \frac{d \hat{\mathrm{v}}}{d t} \\
& =\frac{q}{m \gamma}\left[\left\{\vec{S} \times\left[\vec{B}+G\left(\gamma \vec{B}_{\perp}+\vec{B}_{\|}\right)\right] \cdot \hat{\mathrm{v}}+\vec{S} \cdot \hat{\mathrm{v}} \times \vec{B}\right\} \hat{\mathrm{v}}+(\hat{\mathrm{v}} \cdot \vec{S}) \hat{\mathrm{v}} \times \vec{B}\right] \\
& =\frac{q}{m \gamma}\left\{\left(\vec{S} \times\left[G\left(\gamma \vec{B}_{\perp}+\vec{B}_{\|}\right)\right] \cdot \hat{\mathrm{v}}\right) \hat{\mathrm{v}}+(\hat{\mathrm{v}} \cdot \vec{S}) \hat{\mathrm{v}} \times \vec{B}\right\} \\
& =\frac{q}{m \gamma}\left[\vec{S}_{\|} \times \vec{B}_{\perp}+G \gamma\left(\vec{S}_{\perp} \times \vec{B}_{\perp} \cdot \hat{\mathrm{v}}\right) \hat{\mathrm{v}}\right]
\end{aligned}
$$

and

$$
\frac{d \vec{S}_{\perp}}{d t}=\frac{q}{m \gamma}\left\{\vec{S}_{\perp} \times\left[\vec{B}+G\left(\gamma \vec{B}_{\perp}+\vec{B}_{\|}\right)\right]+G \gamma \vec{S}_{\|} \times \vec{B}_{\perp}-G \gamma\left(\vec{S}_{\perp} \times \vec{B}_{\perp} \cdot \hat{\mathrm{v}}\right) \hat{\mathrm{v}}\right\}
$$


The Thomas-BMT equation (1.1) is independent of the coordinate system. Since all the particles move in the vicinity of a closed orbit, it is convenient to use a coordinate system based on a closed reference orbit as we consider particles whose motion takes place near (though not exactly on) that orbit. We assume the reference orbit is in a plane and has a circumference we denote by $2 \pi R$. We introduce a coordinate system (a Frenet-Serret system) based on this reference orbit. The position of a particle is characterized by the vector $\vec{\xi}$ from the point on the reference orbit closest to the particle, and we define the coordinates to be:

$s=$ the distance along the reference orbit from an origin point (arbitrarily chosen) on the reference orbit to the point on the reference orbit closest to the particle.

$z=$ the vertical component of $\vec{\xi}$, i.e. the distance from the plane of the reference orbit to the particle.

$x=$ the horizontal component of $\vec{\xi}$, which is the length of the projection of $\vec{\xi}$ on the orbit plane. The basis vectors $\hat{e}_{x}, \hat{e}_{s}, \hat{e}_{z}$ are unit vectors in the $x, s$ and $z$ directions. The sign convention is that if the reference orbit is counter-clockwise, $x$ is positive in the plane outside the reference orbit and $z$ is positive above the reference orbit.

We also define $\rho(s)$ to be the radius of curvature of the reference orbit at $s$; in a straight section the curvature $1 / \rho(s)$ is zero, and the coordinates are locally Cartesian.

It is convenient to change to $s$ instead of the time $t$ as the independent variable, with

$$
d s=\frac{\mathrm{v}}{1+x / \rho} d t
$$

(note that $s$ is the distance along the reference orbit, not exactly the distance traversed by the particle).

In what follows we shall use the prime for differentiation by $s$; i.e.

$$
X^{\prime} \equiv \frac{d X}{d s}
$$

for any variable $X$.

We define a reference-orbit based frame with the unit vectors in the $x, s, z$ directions as basis vectors. These basis vectors form a right-handed system. They rotate with s: 


$$
\left(\begin{array}{l}
\hat{e}_{x}^{\prime} \\
\hat{e}_{s}^{\prime} \\
\hat{e}_{z}^{\prime}
\end{array}\right)=\left(\begin{array}{ccc}
0 & 1 / \rho & 0 \\
-1 / \rho & 0 & 0 \\
0 & 0 & 0
\end{array}\right)\left(\begin{array}{l}
\hat{e}_{x} \\
\hat{e}_{s} \\
\hat{e}_{z}
\end{array}\right)
$$

\section{Trajectory-based Frame}

The particles do not necessarily travel on the reference orbit, therefore the spin components in the directions $\hat{e}_{x}, \hat{e}_{s}, \hat{e}_{z}$ are not exactly in the directions transverse and longitudinal to the motion of a particle. Since the dynamical equations (1.3) and (1.4) show that the transverse and longitudinal spin components behave differently, it is desirable to formulate equations of motion that maintain this distinction, i.e. describe, for a given particle, the behavior of the components of spin parallel and perpendicular to the direction of motion of that particle, rather than the components parallel and perpendicular to the reference orbit.

Following Kondratenko and Sivers [3] we introduce a "natural" or "local" reference frame based on the actual trajectory of the particle. The basis vector $\hat{u}_{2}$ is taken to be the unit vector $\hat{\mathrm{v}}=\left(\hat{e}_{s}+x^{\prime} \hat{e}_{x}+z^{\prime} \hat{e}_{z}\right) / \sqrt{1+x^{\prime 2}+z^{\prime 2}}$ in the direction of the instantaneous particle velocity, and the other two are in the local radial and vertical direction orthogonal to $\hat{v}$ and to each other. In what follows we ignore second and higher terms in the excursions $x$ and $z$ of the particle from the reference orbit. The local frame is then based on the unit vectors

$$
\left(\begin{array}{l}
\hat{u}_{1} \\
\hat{u}_{2} \\
\hat{u}_{3}
\end{array}\right)=\left(\begin{array}{ccc}
1 & -x^{\prime} & 0 \\
x^{\prime} & 1 & z^{\prime} \\
0 & -z^{\prime} & 1
\end{array}\right)\left(\begin{array}{l}
\hat{e}_{x} \\
\hat{e}_{s} \\
\hat{e}_{z}
\end{array}\right) ; \text { thus }=\left(\begin{array}{l}
\hat{e}_{x} \\
\hat{e}_{s} \\
\hat{e}_{z}
\end{array}\right)=\left(\begin{array}{ccc}
1 & x^{\prime} & 0 \\
-x^{\prime} & 1 & -z^{\prime} \\
0 & z^{\prime} & 1
\end{array}\right)\left(\begin{array}{l}
\hat{u}_{1} \\
\hat{u}_{2} \\
\hat{u}_{3}
\end{array}\right)
$$

The new basis vectors, of course, also rotate; using (1.6) and (2.1) we obtain, to first order in $x$ and $z$ and their derivatives 


$$
\begin{aligned}
\left(\begin{array}{l}
\hat{u}_{1}{ }^{\prime} \\
\hat{u}_{2}{ }^{\prime} \\
\hat{u}_{3}^{\prime}
\end{array}\right) & =\left[\left(\begin{array}{ccc}
0 & -x^{\prime \prime} & 0 \\
x^{\prime \prime} & 0 & z^{\prime \prime} \\
0 & -z^{\prime \prime} & 0
\end{array}\right)+\left(\begin{array}{ccc}
1 & -x^{\prime} & 0 \\
x^{\prime} & 1 & z^{\prime} \\
0 & -z^{\prime} & 1
\end{array}\right)\left(\begin{array}{ccc}
0 & 1 / \rho & 0 \\
-1 / \rho & 0 & 0 \\
0 & 0 & 0
\end{array}\right)\right]\left(\begin{array}{l}
\hat{e}_{x} \\
\hat{e}_{s} \\
\hat{e}_{z}
\end{array}\right) \\
& =\left(\begin{array}{ccc}
x^{\prime} / \rho & 1 / \rho-x^{\prime \prime} & 0 \\
-1 / \rho+x^{\prime \prime} & x^{\prime} / \rho & z^{\prime \prime} \\
z^{\prime} / \rho & -z^{\prime \prime} & 0
\end{array}\right)\left(\begin{array}{ccc}
1 & x^{\prime} & 0 \\
-x^{\prime} & 1 & -z^{\prime} \\
0 & z^{\prime} & 1
\end{array}\right)\left(\begin{array}{l}
\hat{u}_{1} \\
\hat{u}_{2} \\
\hat{u}_{3}
\end{array}\right) \\
& =\left(\begin{array}{ccc}
0 & 1 / \rho-x^{\prime \prime} & -z^{\prime} / \rho \\
-1 / \rho+x^{\prime \prime} & 0 & z^{\prime \prime} \\
z^{\prime} / \rho & -z^{\prime \prime} & 0
\end{array}\right)\left(\begin{array}{l}
\hat{u}_{1} \\
\hat{u}_{2} \\
\hat{u}_{3}
\end{array}\right)
\end{aligned}
$$

Note that the rotations of the basis vectors in the "natural" frame are more complicated than those in the reference-orbit based frame (1.6).

Note that the independent variable $s$ and the excursions $x$ and $z$ are still defined with respect to the reference orbit, while the basis vectors are derived from the actual trajectory.

With $s$ as the independent variable equation (1.1) becomes

$$
\vec{S}^{\prime} \equiv \frac{d \vec{S}}{d s}=\frac{1+x / \rho}{\mathrm{v}} \frac{d \vec{S}}{d t}=\vec{S} \times \vec{F} ; \quad \vec{F}=\frac{1+x / \rho}{B \rho}\left[\vec{B}+G\left(\gamma \vec{B}_{\perp}+\vec{B}_{\|}\right)\right]
$$

where $B \rho=\frac{m \gamma \mathrm{v}}{q}$ is the magnetic rigidity of the particle, so that in terms of the basis vectors $\hat{u}_{1}, \hat{u}_{2}$ and $\hat{u}_{3}$ we can write

$$
\left(\begin{array}{l}
S_{1}{ }^{\prime} \\
S_{2}{ }^{\prime} \\
S_{3}{ }^{\prime}
\end{array}\right)=\left(\begin{array}{l}
\vec{S} \times \vec{F} \cdot \hat{u}_{1}+\vec{S} \cdot \hat{u}_{1}{ }^{\prime} \\
\vec{S} \times \vec{F} \cdot \hat{u}_{2}+\vec{S} \cdot \hat{u}_{2}{ }^{\prime} \\
\vec{S} \times \vec{F} \cdot \hat{u}_{3}+\vec{S} \cdot \hat{u}_{3}{ }^{\prime}
\end{array}\right)=\vec{S} \times \vec{W}
$$

with

$$
\vec{W}=\vec{F}+z^{\prime \prime} \hat{u}_{1}+\frac{z^{\prime}}{\rho} \hat{u}_{2}+\left(\frac{1}{\rho}-x "\right) \hat{u}_{3}
$$

It is convenient to express this in terms of the excursions of the particle. Courant and Ruth [4] and Lee [5] express $\vec{B}_{\perp}, \vec{B}_{\|}$and $\vec{F}$ in terms of the particle excursions, governed by the Lorentz force equation (1.2). In terms of the fixed vectors $\hat{e}$ 


$$
\begin{aligned}
& \vec{B}_{\perp}=B \rho\left(1-\frac{x}{\rho}\right)\left[\left(x "-\frac{1}{\rho}\right) \hat{e}_{z}+\frac{z^{\prime}}{\rho} \hat{e}_{s}-z^{\prime \prime} \hat{e}_{x}\right] \\
& \vec{B}_{\|}=\left[B_{s o l}-B \rho\left(\frac{z}{\rho}\right)^{\prime}\right] \hat{e}_{s}
\end{aligned}
$$

where $B_{s o l}$ is the solenoidal field on the reference orbit, which was not included in [4] and [5].

In the trajectory-based coordinate system (2.1) this becomes

$$
\begin{aligned}
& \vec{B}_{\perp}=B \rho\left[\left(x "-\frac{1}{\rho}+\frac{x}{\rho^{2}}\right) \hat{u}_{3}-z^{\prime \prime} \hat{u}_{1}\right] \\
& \vec{B}_{\|}=\left[B_{s o l}-B \rho\left(\frac{z}{\rho}\right)^{\prime}\right] \hat{u}_{2}
\end{aligned}
$$

Note that $\vec{B}_{\perp}$ has no component in the direction $\hat{u}_{2}$, confirming the validity of the division into transverse and longitudinal components.

We thus have (again to first order in the displacements $x$ and $z$ and their derivatives)

$$
\begin{aligned}
& \vec{F}=-(1+G \gamma) z^{\prime \prime} \hat{u}_{1}+(1+G)\left[\frac{B_{\text {sol }}}{B \rho}-\left(\frac{z}{\rho}\right)^{\prime}\right] \hat{u}_{2}-(1+G \gamma)\left(\frac{1}{\rho}-x^{\prime \prime}\right) \hat{u}_{3} \\
& \vec{W}=-G \gamma z^{\prime \prime} \hat{u}_{1}+\left\{(1+G)\left[\frac{B_{s o l}}{B \rho}-z\left(\frac{1}{\rho}\right)^{\prime}\right]-G \frac{z^{\prime}}{\rho}\right\} \hat{u}_{2}-G \gamma\left(\frac{1}{\rho}-x^{\prime \prime}\right) \hat{u}_{3}
\end{aligned}
$$

and expressing (2.4) in terms of the coordinates gives

$$
\begin{aligned}
& S_{1}{ }^{\prime}=-S_{2} G \gamma\left(\frac{1}{\rho}-x^{\prime \prime}\right)-S_{3}\left\{(1+G)\left[\frac{B_{s o l}}{B \rho}-z\left(\frac{1}{\rho}\right)^{\prime}\right]-G \frac{z^{\prime}}{\rho}\right\} \\
& S_{2}{ }^{\prime}=G \gamma\left[-S_{3} z^{\prime \prime}+S_{1}\left(\frac{1}{\rho}-x^{\prime \prime}\right)\right] . \\
& S_{3}{ }^{\prime}=S_{1}\left\{(1+G)\left[\frac{B_{\text {sol }}}{B \rho}-z\left(\frac{1}{\rho}\right)^{\prime}\right]-G \frac{z^{\prime}}{\rho}\right\}+S_{2} G \gamma z^{\prime \prime}
\end{aligned}
$$


Note that if $G=0$, i.e. if there is no anomalous magnetic moment, the longitudinal spin component $S_{2}$ is constant: helicity is conserved.

The dominant terms in the equations for $S_{1}{ }^{\prime}$ and $S_{2}{ }^{\prime}$ are $\mp G \gamma / \rho$, leading to the precession frequency (spin tune) $\mathrm{G} \gamma$. 


\section{Spinor Formalism, Depolarizing Resonances}

Spin dynamics is conveniently formulated with the use of spinor (SU2) algebra. We introduce a 2-component spinor

$$
\Psi=\left(\begin{array}{c}
\Psi_{1} \\
\Psi_{2}
\end{array}\right)
$$

whose property is that the components of the spin $\vec{S}$ are

$$
S_{i}=<\Psi\left|\sigma_{i}\right| \Psi>=\Psi^{\dagger} \sigma_{i} \Psi
$$

with the Pauli matrices $\sigma_{1}=\left(\begin{array}{ll}0 & 1 \\ 1 & 0\end{array}\right), \sigma_{2}=\left(\begin{array}{cc}0 & -i \\ i & 0\end{array}\right), \sigma_{3}=\left(\begin{array}{cc}1 & 0 \\ 0 & -1\end{array}\right)$

so that the equation of motion (2.4) for the components $S_{i}$ is equivalent to the spinor equation

$$
\begin{aligned}
\Psi^{\prime} & =-\frac{i}{2}(\vec{\sigma} \cdot \vec{W}) \Psi=-\frac{i}{2}\left(\begin{array}{cc}
W_{3} & W_{1}-i W_{2} \\
W_{1}+i W_{2} & -W_{3}
\end{array}\right) \Psi \\
& =-\frac{i}{2}\left(\begin{array}{cc}
G \gamma\left(x^{\prime \prime}-1 / \rho\right) & \zeta \\
\varsigma^{*} & -G \gamma\left(x^{\prime \prime}-1 / \rho\right)
\end{array}\right) \Psi
\end{aligned}
$$

with $W$ defined by $(2.8)$, and

$$
\zeta=W_{1}-i W_{2}=-G \gamma z^{\prime \prime}+i\left[G z^{\prime} \rho+(1+G) z(1 / \rho)^{\prime}-(1+G) B_{s o l} / B \rho\right]
$$

We now transform the spinor to

$$
\Phi=\exp \left(-\frac{i}{2} \sigma_{3} \chi\right) \Psi
$$

with

$$
\theta=s / R ; \quad \Theta=\int_{0}^{s} d s^{\prime} / \rho-x^{\prime} ; \quad \chi=G \gamma(\theta-\Theta)
$$

$\Theta$ is the turning angle; $\theta$ is the azimuth along the reference orbit. Then 


$$
\begin{aligned}
\Phi^{\prime} & =-\frac{i}{2}\left\{G \gamma \sigma_{3}\left(\frac{1}{R}-\frac{1}{\rho}+x "\right)+e^{-\frac{i}{2} \chi \sigma_{3}}\left[G \gamma\left(\frac{1}{\rho}-x "\right) \sigma_{3}+W_{1} \sigma_{1}+W_{2} \sigma_{2}\right] e^{\frac{i}{2} \chi \sigma_{3}}\right\} \Phi \\
& =-\frac{i}{2}\left(\begin{array}{cc}
G \gamma / R & \zeta e^{-i \chi} \\
\zeta^{*} e^{i \chi} & -G \gamma / R
\end{array}\right) \Phi
\end{aligned}
$$

The diagonal elements of the matrix in (3.7) tell us that the basic precession frequency about the vertical (spin tune) is $G \gamma$, and the off-diagonal terms produce changes (depolarization) in the spin. The depolarizing term $\zeta e^{-i \chi}$ is a combination of oscillations at various frequencies. Therefore it may be written in the form

$$
\zeta e^{-i \chi}=\frac{1}{R} \sum_{r} \varepsilon_{r} e^{-i \kappa_{r} \theta}
$$

where resonances occur at the frequencies $\kappa_{r}$ and $\varepsilon_{r}$ is the strength of the resonance at $G \gamma=$ $\kappa_{r}$. Here $\kappa_{r}$, the $r$-th resonance value of $G \gamma$, may be

Imperfection resonances: $\kappa_{r}=$ an integer $k$, for imperfection resonances.

"Intrinsic" resonances (due to vertical betatron oscillations): $\kappa_{r}=k P \pm v_{z}$, where $v_{z}$ is the vertical betatron tune, $P$ is the periodicity of the magnet structure, $k$ is any integer.

Broken periodicity resonances $\kappa_{r}=k \pm v_{z}$ occur when the structure periodicity $P$ is inexact.

$\mathrm{RF}$ resonances $\kappa_{r}=k \pm \omega_{r f} / \omega_{\text {orbit }}$ induced by radiofrequency dipoles and/or solenoids placed somewhere on the orbit. These will be treated in section 5 of this report.

In this report we omit discussions of:

Horizontal resonances $\kappa_{r}=k \pm v_{x}$ due to the fact that horizontal betatron oscillations produce turning angles per revolution that are not exactly $2 \pi$;

Horizontal orbit excursions;

Higher order resonances. 
When the orbit is the closed orbit produced by imperfections, without betatron oscillations, (3.8) is a straightforward Fourier series and resonances occur when $G \gamma=$ an integer $k$, the resonance strengths $\varepsilon_{k}$ are calculated by Fourier analysis of $\zeta e^{-i \chi}$ as defined in (3.4):

$$
\varepsilon_{k}=\frac{1}{2 \pi} \int_{0}^{2 \pi R} \zeta e^{i(k \theta-\chi)} d s
$$

If betatron oscillations are present we have intrinsic and/or broken periodicity resonances $\kappa_{r}=k \pm v_{z}$; we consider $z$ to be the trajectory of betatron oscillations of frequency $v_{z}$. The frequencies $\kappa_{i}$ in (3.8) are not multiples of one frequency; therefore (3.8) is no longer a simple Fourier series but a more general combination of oscillations (an almost periodic function), and Fourier analysis (3.9) does not apply. Instead we note that for $G \gamma=$ any resonance frequency $\kappa$ the component of frequency $\kappa$ of the trigonometric series is

$$
\varepsilon_{\kappa}=\lim _{n \rightarrow \infty}\left(\frac{1}{4 n \pi} \int_{-2 n \pi R}^{2 n \pi R} \zeta e^{i(\kappa \theta-\chi)} d s\right)=\left\langle\frac{1}{2 \pi} \int_{2 n \pi R}^{2(n+1) \pi R} \zeta e^{i(\kappa \theta-\chi)} d s\right\rangle
$$

where the angular brackets denote the mean over all $n$. But since $z=z_{-}(\theta) e^{-i v_{z} \theta}+z_{+}(\theta) e^{i v_{z} \theta}$, where $z_{+}(\theta)$ and $z_{-}(\theta)=z_{+}{ }^{*}(\theta)$ are periodic functions, we can separate $\zeta$ into $\zeta_{+}+\zeta_{-}$and perform Fourier analysis for each part, and find

$$
\begin{aligned}
& \text { for } G \gamma=\kappa=k+v_{z}: \varepsilon_{\kappa}=\frac{1}{2 \pi} \int_{0}^{2 \pi R} \zeta_{-} e^{i(\kappa \theta-\chi)} d s=\frac{1}{2 \pi} \int_{0}^{2 \pi R} \zeta_{-} e^{i G \gamma \Theta} d s \\
& \text { for } G \gamma=\kappa=k-v_{z}: \varepsilon_{\kappa}=\frac{1}{2 \pi} \int_{0}^{2 \pi R} \zeta_{+} e^{i(\kappa \theta-\chi)} d s=\frac{1}{2 \pi} \int_{0}^{2 \pi R} \zeta_{+} e^{i G \gamma \Theta} d s
\end{aligned}
$$

To evaluate the resonance strengths in any particular case it is necessary to express the integration factors $\zeta, \zeta_{+}, \zeta_{-}$in (3.9), (3.10), and (3.11) in terms of the components of the lattice structure, as is done in [4].

\section{Comparison with Reference-Orbit Frame}

In the "reference orbit" frame used in most of the literature ([4], [5]. [6]) the equation for the components of $\vec{S}$, analogous to (2.4) is 


$$
\left(\begin{array}{l}
S_{x}{ }^{\prime} \\
S_{s}{ }^{\prime} \\
S_{z}{ }^{\prime}
\end{array}\right)=\left(\begin{array}{l}
\vec{S} \times \vec{F} \cdot \hat{e}_{x}+\vec{S} \cdot \hat{e}_{x}{ }^{\prime} \\
\vec{S} \times \vec{F} \cdot \hat{e}_{s}+\vec{S} \cdot \hat{e}_{s}{ }^{\prime} \\
\vec{S} \times \vec{F} \cdot \hat{e}_{z}+\vec{S} \cdot \hat{e}_{z}{ }^{\prime}
\end{array}\right)=\vec{S} \times \vec{E}
$$

with

$$
\vec{E}=\vec{F}+\hat{e}_{z} / \rho
$$

With $\vec{F}$ expressed in terms of the reference frame (equations (2.8) and (2.6)) we have

$$
\vec{E}=G \gamma\left(x^{\prime \prime}-\frac{1}{\rho}\right) \hat{e}_{z}+\left[(1+G \gamma) z^{\prime} / \rho-(1+G)(z / \rho)^{\prime}+(1+G) \frac{B_{s o l}}{B \rho}\right] \hat{e}_{s}-(1+G \gamma) z^{\prime \prime} \hat{e}_{x}
$$

Going through the same steps as in the previous section, we arrive at the spinor equation analogous to (3.3):

$$
\begin{aligned}
\Phi^{\prime} & =-\frac{i}{2}\left\{\frac{G \gamma}{R} \sigma_{3}+\left(E_{x} \sigma_{1}+E_{s} \sigma_{2}\right) \exp \left[i \chi \sigma_{3}\right]\right\} \Phi \\
& =-\frac{i}{2}\left(\begin{array}{cc}
\frac{G \gamma}{R} & \zeta^{(e)} e^{-i \chi} \\
\zeta^{(e)^{*}} e^{i \chi} & -\frac{G \gamma}{R}
\end{array}\right) \Phi \\
\zeta^{(e)} & =E_{x}-i E_{s}=-(1+G \gamma) z^{\prime \prime}-i\left[(1+G \gamma) z^{\prime} / \rho-(1+G)(z / \rho)^{\prime}+(1+G) \frac{B_{\text {sol }}}{B}\right] \\
& =-(1+G \gamma) z^{\prime \prime}-i\left[G(\gamma-1) z^{\prime} / \rho-(1+G) z(1 / \rho)^{\prime}+(1+G) \frac{B_{s o l}}{B}\right]
\end{aligned}
$$

and the resonance strength is

$$
\varepsilon_{\kappa}^{(e)}=\left\langle\frac{1}{2 \pi} \int_{2 n \pi R}^{2(n+1) \pi R} \zeta^{(e)} e^{i(\kappa \theta-\chi)} d s\right\rangle
$$

Note that the leading term $-(1+G \gamma) z^{\prime \prime}$ in $\zeta^{(e)}$ is different from the leading term $-G \gamma z "$ in $\zeta$, which makes it plausible to surmise that resonance strengths in the two frames differ by the factor $G \gamma /(1+G \gamma)$ as indicated in [7]. But this is not the case, as shown by Kondratenko [3]. His derivation, in our terminology, is:

The difference between the resonance strength calculated in the two different frames is 


$$
\varepsilon_{\kappa}-\varepsilon_{\kappa}^{(e)}=\left\langle\frac{1}{2 \pi} \int_{2 n \pi R}^{2(n+1) \pi R}\left(\zeta-\zeta^{(e)}\right) e^{i(\kappa \theta-\chi)} d s\right\rangle
$$

and

$$
\begin{aligned}
\zeta-\zeta^{(e)} & =W_{1}-E_{x}-i\left(W_{2}-E_{s}\right) \\
& =z^{\prime \prime}+i G \gamma z^{1 / \rho}
\end{aligned}
$$

At the resonance $\kappa=G \gamma$, we have $\kappa \theta-\chi=G \gamma \theta-G \gamma(\theta-\Theta)=G \gamma \Theta$, and the integrand of (4.6) is

$$
\left(\zeta-\zeta^{(e)}\right) e^{i G \xi \Theta}=\left[z^{\prime \prime}+i G \gamma \Theta^{\prime} z^{\prime}\right] e^{i G \gamma \Theta}=\frac{d}{d s}\left(z^{\prime} e^{i G \gamma \Theta}\right)
$$

That is a perfect derivative. The integral of a perfect derivative over a period averages to zero. Therefore $\varepsilon_{K}=\varepsilon_{K}{ }^{(e)}$ : the resonance strength is independent of the frame in which it is calculated.

It follows that in any particular case where the strengths of resonances are to be calculated one may select the trajectory- based formalism of section 3 or the reference-orbit formalism of section 4, whichever is more convenient. The algorithms given in [4] for calculation of resonance strengths for a given magnet structure, leading to the computer program DEPOL, remain valid.

\section{Resonance Strength with RF Solenoids or Dipoles}

RF solenoids or dipoles may be inserted in an accelerator or storage ring in order to deliberately excite spin resonances, either for the purpose of reversing (flipping) the spin, or to enhance intrinsic resonances to a strength where the spin reverses completely rather than partially.

The strength of these induced resonances may be calculated by using the results of the previous sections.

\section{5a. RF Solenoids}

First; consider a radiofrequency solenoid with field $B_{s o l}=\sqrt{2} B_{r m s} \cos \omega_{r f} t$.at one point in the ring, which we designate as $\theta=0$. It rotates the spin by an angle

$$
\Delta_{s} \cos \omega_{r f} t ; \Delta_{s}=(1+G) \frac{\int \sqrt{2} B_{r m s} d s}{B \rho}
$$


once per revolution, and does not affect the orbit. Therefore we may set $z=z^{\prime}=0$ in sections 3 and 4 , and (3.4) simplifies to

$$
\zeta=-i(1+G) B_{s o l} / B \rho=-i \Delta_{s} \delta_{p}(\theta) \cos \nu_{r f} \theta
$$

where $\delta_{p}(\theta)$ is the periodic delta function and $v_{r f}=\omega_{r f} / \omega_{\text {orbit }}$ is the rf frequency normalized to the revolution frequency. Since $\delta_{p}(\theta)=\frac{1}{2 \pi} \sum_{k=-\infty}^{\infty} e^{i k \theta}$ and $\cos v_{r f} \theta=\frac{1}{2}\left(e^{i v_{r f} \theta}+e^{-i v_{r f} \theta}\right)$ we can, as in Section 3, divide $\zeta$ into $\zeta_{+}+\zeta_{-}$and obtain

$$
\begin{aligned}
& \text { for } G \gamma=\kappa=k+v_{r f}: \varepsilon_{\kappa}=\frac{1}{2 \pi R} \int_{0}^{2 \pi R} \zeta_{-} e^{i \kappa \theta} d s=-\frac{i}{4 \pi} \Delta_{s}=-\frac{i(1+G)}{4 \pi} \frac{\int \sqrt{2} B_{r m s} d s}{B \rho} \\
& \text { for } G \gamma=\kappa=k-v_{r f}: \varepsilon_{\kappa}=\frac{1}{2 \pi R} \int_{0}^{2 \pi R} \zeta_{+} e^{i \kappa \theta} d s=-\frac{i}{4 \pi} \Delta_{s}=-\frac{i(1+G)}{4 \pi} \frac{\int \sqrt{2} B_{r m s} d s}{B \rho}
\end{aligned}
$$

in agreement with [6].

Thus there are two resonances of equal strength and equal phase in each interval of $\kappa=G \gamma$ between one integer and the next. If $v_{r f}$ is exactly a half integer these coalesce into a single resonance of twice the strength.

Note that, since $\Delta_{s}$ contains the factor $1 / B \rho$ these resonances become weak at high energy; therefore rf solenoids are primarily useful for low-energy rings such as IUCF.

\section{5b. RF Dipoles}

An alternative is to use rf dipoles with transverse fields. We assume that we have a radiofrequency dipole with radial horizontal field $\sqrt{2} B_{r m s} \cos \omega_{r f} t$ at one point in the ring, which we designate as $\theta=0$. The beam deflection it produces is

$$
\Delta \cos \omega_{r f} t ; \quad \Delta=\frac{\int \sqrt{2} B_{r m s} d s}{B \rho}
$$

The spin rotation associated with this deflection is, according to eq. (1.1), just $(1+G \gamma)$ times the deflection(5.4), and this leads to the naïve equation

$$
\varepsilon=(1+G \gamma) \frac{\Delta}{4 \pi}=(1+G \gamma) \frac{\int \sqrt{2} B_{r m s} d s}{4 \pi B \rho}
$$


for the resonance strength at $G \gamma=k \pm v_{r f}[6,9]$. But the dipole inevitably induces a forced vertical oscillation in the whole ring, which in turn also affects the spin just like any other vertical oscillation, and this effect must also be considered, as recognized in the text of [9] and by other authors [10].

The equation of orbit motion for the forced oscillations is

$$
\frac{d^{2} z}{d s^{2}}+K(s) z=\frac{\Delta}{R} \delta_{p}(\theta) \cos v_{r f} \theta
$$

where $K(s)$ is the focusing gradient function, $\theta=s / R$ is the normalized azimuth, $\delta_{p}(\theta)$ is the periodic delta function, and $v_{r f}=\omega_{r f} / \omega_{\text {orbit }}$. We assume the solution of the homogeneous equation corresponding to (5.6) is known; the vertical tune is $v_{z}$ and the orbit functions are $\beta_{z}(s), \alpha_{z}(s)$. Transforming to simple harmonic formulation by going to the variables[8]

$$
\eta=\frac{z}{\sqrt{\beta_{z}(s)}} ; \quad \varphi(s)=\frac{1}{v_{z}} \int \frac{d s}{\beta_{z}}
$$

the inhomogeneous equation (5.6) becomes

$$
\frac{d^{2} \eta}{d \varphi^{2}}+v_{z}^{2} \eta=\frac{v_{z}^{2} \beta_{z}^{3 / 2} \Delta}{R} \delta_{p}(\theta) \cos v_{r f} \theta
$$

(Note that there are three different angles that increment by $2 \pi$ every revolution: the normalized azimuth $\theta=s / R, \theta=$ the bending angle $\int d s / \rho$ on the reference orbit, and the betatron phase angle $\varphi$; we chose all three of these angles to be $=0(\bmod 2 \pi)$ at the azimuth of the rf dipole).

Using the relations

$$
\Delta=\frac{\int \sqrt{2} B_{r m s} d s}{B \rho} ; \delta_{p}(\theta)=\frac{R}{v_{z} \beta_{z}} \delta_{p}(\varphi)
$$

and again defining the resonant tune $v_{r f}$ as $v_{r f}=\omega_{r f} / \omega_{o r b i t}$ we end up with

$$
\frac{d^{2} \eta}{d \varphi^{2}}+v_{z}{ }^{2} \eta=v_{z} \beta_{z}{ }^{1 / 2} \Delta \delta_{p}(\varphi) \cos v_{r f} \varphi
$$


Since $\delta_{p}(\varphi)=\frac{1}{2 \pi} \sum_{k=-\infty}^{\infty} e^{i k \varphi} ; \quad \cos v_{r f} \varphi=\frac{1}{2}\left(e^{i v_{r f} \varphi}+e^{-i v_{r f} \varphi}\right)$, (5.9) becomes

$$
\frac{d^{2} \eta}{d \varphi^{2}}+v_{z}^{2} \eta=\frac{v_{z} \beta_{z}^{1 / 2} \Delta}{4 \pi} \sum_{k=-\infty}^{\infty}\left(e^{i\left(k+v_{r}\right) \varphi}+e^{i\left(k-v_{r f}\right) \varphi}\right)
$$

the solution of which is

$$
\begin{aligned}
& \eta=\beta_{z}^{-1 / 2} z=\sum_{k=-\infty}^{\infty}\left(\eta_{k+}+\eta_{k-}\right) ; \\
& \eta_{k+}=\frac{v_{z} \beta_{z}{ }^{1 / 2} \Delta}{4 \pi} \frac{e^{i\left(k+v_{r f}\right) \varphi}}{v_{z}{ }^{2}-\left(k+v_{r f}\right)^{2}} ; \quad \eta_{k-}=\frac{v_{z} \beta_{z}^{1 / 2} \Delta}{4 \pi} \frac{e^{i\left(k-v_{r}\right) \varphi}}{v_{z}{ }^{2}-\left(k-v_{r f}\right)^{2}}
\end{aligned}
$$

The equations of spin dynamics expressed in terms of the trajectory excursion $z(s)$, as described in sections 3 and 4, remain valid. To find the resonance strengths at $G \gamma=k \pm v_{r f}$ we use the trajectory-based frame of section 3 (the alternate frame, section 4 , based on the reference orbit gives identical results). Recall $z=\beta_{z}{ }^{1 / 2} \eta, \zeta=-G \gamma z^{\prime \prime}+i\left[G z^{\prime} / \rho+(1+G) z(1 / \rho)\right]$, and the identities

$$
\begin{aligned}
& \frac{d}{d s}=\frac{1}{v_{z} \beta_{z}} \frac{d}{d \varphi} ; \frac{d^{2}}{d s^{2}}=\frac{2 \alpha_{z}}{v_{z} \beta_{z}^{2}} \frac{d}{d \varphi}+\frac{1}{v_{z}^{2} \beta_{z}^{2}} \frac{d^{2}}{d \varphi^{2}} \\
& \frac{d \beta_{z}}{d s}=-2 \alpha_{z} ; \frac{d \alpha_{z}}{d s}=\frac{K \beta_{z}^{2}-1-\alpha_{z}^{2}}{\beta_{z}}
\end{aligned}
$$

giving

$$
\begin{aligned}
z^{\prime} & =-\frac{\alpha_{z}}{\beta_{z}^{1 / 2}} \eta+\frac{1}{v_{z} \beta_{z}^{1 / 2}} \frac{d \eta}{d \varphi}=-\frac{1}{\beta_{z}^{1 / 2}}\left(\alpha_{z} \eta-\frac{1}{v_{z}} \frac{d \eta}{d \varphi}\right) \\
z^{\prime \prime} & =-\frac{\alpha_{z}}{\beta^{3 / 2}}\left(\alpha_{z} \eta-\frac{1}{v_{z}} \frac{d \eta}{d \varphi}\right)-\frac{\left(K \beta^{2}-1-\alpha_{z}^{2}\right) \eta}{\beta_{z}^{3 / 2}}-\frac{\alpha_{z}}{v_{z} \beta_{z}^{3 / 2}} \frac{d \eta}{d \varphi}+\frac{1}{v_{z}^{2} \beta_{z}^{3 / 2}} \frac{d^{2} \eta}{d \varphi^{2}} \\
& =\frac{1}{\beta_{z}^{3 / 2}}\left[\left(1-K \beta_{z}{ }^{2}\right) \eta+\frac{1}{v_{z}{ }^{2}} \frac{d^{2} \eta}{d \varphi^{2}}\right]
\end{aligned}
$$

Using the reference-orbit coordinate system of section 4 we have 


$$
\begin{aligned}
\zeta^{(e)} & =-(1+G \gamma) z^{\prime \prime}-i\left[G(\gamma-1) z^{\prime} / \rho-(1+G) z(1 / \rho)^{\prime}\right] \\
& =\frac{1+G \gamma}{\beta_{z}^{3 / 2}}\left[\left(K \beta_{z}{ }^{2}-1\right) \eta-\frac{1}{v_{z}^{2}} \frac{d^{2} \eta}{d \varphi^{2}}\right]+i \frac{G(\gamma-1)}{\rho \beta_{z}^{1 / 2}}\left(\alpha_{z} \eta-\frac{1}{v_{z}} \frac{d \eta}{d \varphi}\right)+i(1+G) z(1 / \rho)^{\prime}
\end{aligned}
$$

Each term of the trigonometric series (5.11) is an oscillation of frequency $k-v_{r f}$ or $k+v_{r f}$, therefore we can again, as before, separate $\zeta^{(e)}$ into $\zeta_{+}+\zeta_{-}$:

$$
\begin{aligned}
& \zeta^{(e)}=\frac{1+G \gamma}{\beta_{z}^{3 / 2}}\left[\left(K \beta_{z}{ }^{2}-1\right) \eta-\frac{1}{v_{z}{ }^{2}} \frac{d^{2} \eta}{d \varphi^{2}}\right]+i \frac{G(\gamma-1)}{\rho \beta_{z}^{1 / 2}}\left(\alpha_{z} \eta-\frac{1}{v_{z}} \frac{d \eta}{d \varphi}\right)+i(1+G) z(1 / \rho)^{\prime} \\
& \zeta_{+}=\frac{v_{z} \Delta}{4 \pi} \sum_{k=-\infty}^{\infty} \frac{e^{i \kappa_{+} \varphi}}{v_{z}{ }^{2}-\kappa_{+}^{2}}\left[\frac{1+G \gamma}{\beta_{z}}\left(K \beta_{z}{ }^{2}-1+\frac{\kappa_{+}{ }^{2}}{v_{z}^{2}}\right)+\frac{G(\gamma-1)\left(i \alpha_{z}+\kappa_{+} / v_{z}\right)}{\rho}+i(1+G) \beta_{z}(1 / \rho)^{\prime}\right] \\
& \zeta_{-}=\frac{v_{z} \Delta}{4 \pi} \sum_{k=-\infty}^{\infty} \frac{e^{i \kappa_{-} \varphi}}{v_{z}{ }^{2}-\kappa_{+}{ }^{2}}\left[\frac{1+G \gamma}{\beta_{z}}\left(K \beta_{z}{ }^{2}-1+\frac{\kappa_{-}^{2}}{v_{z}^{2}}\right)+\frac{G(\gamma-1)\left(i \alpha_{z}+\kappa_{-} / v_{z}\right)}{\rho}+i(1+G) \beta_{z}(1 / \rho)^{\prime}\right] \\
& \text { where } \kappa_{+}=k+v_{r f}, \quad \kappa_{-}=k-v_{r f}
\end{aligned}
$$

The resonance strengths are, analogously with (3.10)

$$
\begin{aligned}
& \text { for } G \gamma=\kappa=n+v_{r f}: \\
& \varepsilon_{\kappa}=\frac{1}{2 \pi} \int_{0}^{2 \pi R} \zeta_{-} e^{i \kappa \varphi} d s=\frac{v_{z}}{2 \pi} \int_{0}^{2 \pi} \beta_{z} \zeta_{-} e^{i \kappa \varphi} d \varphi \\
& \text { for } G \gamma=\kappa=n-v_{r f}: \\
& \varepsilon_{\kappa}=\frac{1}{2 \pi} \int_{0}^{2 \pi R} \zeta_{+} e^{i \kappa \varphi} d s=\frac{v_{z}}{2 \pi} \int_{0}^{2 \pi} \beta_{z} \zeta_{+} e^{i \kappa \varphi} d \varphi
\end{aligned}
$$

In the simple case of uniform focusing, $\beta_{z}=R / \nu_{z} ; \alpha_{z}=0 ; K \beta_{z}{ }^{2}=1 ; \rho=R ; \varphi=\theta,(1 / \rho)^{\prime}=0$ and (5.15) becomes

$$
\begin{aligned}
& \zeta_{+}=\frac{\Delta}{4 \pi R} \sum_{k=-\infty}^{\infty} \frac{e^{i \kappa_{+} \varphi}}{v_{z}{ }^{2}-\kappa_{+}{ }^{2}}\left[(1+G \gamma) \kappa_{+}^{2}+G(\gamma-1) \kappa_{+}\right] \\
& \zeta_{-}=\frac{\Delta}{4 \pi R} \sum_{k=-\infty}^{\infty} \frac{e^{i \kappa_{-} \varphi}}{v_{z}{ }^{2}-\kappa_{-}{ }^{2}}\left[(1+G \gamma) \kappa_{-}^{2}+G(\gamma-1) \kappa_{-}\right]
\end{aligned}
$$

and for each $\mathrm{k}$ only a single term of the infinite series contributes to (5.16): For $\kappa=G \gamma=n+v_{r f}$ (n any integer) this is the term $k=-n, \kappa_{-}=-n-v_{r f}=-G \gamma$ in $\zeta_{-}$, obtaining 


$$
\begin{aligned}
& \zeta_{\kappa-}=\frac{\Delta}{4 \pi R} \frac{e^{-i G \gamma \varphi}}{V_{z}^{2}-(G \gamma)^{2}}\left[(1+G \gamma)(G \gamma)^{2}-G(\gamma-1) G \gamma\right] \\
& \varepsilon_{\kappa}=\frac{R}{2 \pi} \int_{0}^{2 \pi} \zeta_{\kappa-} e^{i G \gamma \varphi} d \varphi=\frac{\int \sqrt{2} B_{r m s} d s}{4 \pi B \rho} \frac{(G \gamma)^{3}+G^{2} \gamma}{v_{z}^{2}-(G \gamma)^{2}}
\end{aligned}
$$

and the same expression for $\kappa=G \gamma=n-v_{r f}$, in agreement with Kondratenko, Kondratenko and Filatov [11]. 


\section{Acknowledgements}

This work had its origin in a series of telephone conferences led by A D Krisch. The author has benefited from discussions with participants in these conferences and others, in particular A W Chao, H Huang, A M Kondratenko, A D Krisch, M A Leonova, A U Luccio, W MacKay, S R Mane, V A Morozov, Y F Orlov, T Roser, D W Sivers.

\section{References}

[1] M Froissart and R Stora, Nucl. Inst. Meth. 7, 297 (1960)

[2] L H Thomas, Phil. Mag., Series 7, vol. 3, \#13 (1927); V Bargmann, L Michel, V Telegdi, $P R L$ 2, 435 (1959)

[3] A M Kondratenko, contributions to Michigan-COSY teleconferences 6/21/02 and 9/13/07; D W Sivers et al, contributions to Michigan-COSY teleconferences 3/20/07, 4/19/07 and $8 / 9 / 07$

[4] E D Courant and R D Ruth, BNL Report BNL-51270 (1980)

[5] S Y Lee, Spin Dynamics and Snakes in Synchrotrons (World Scientific,1997), Chapters 2,3

[6] A W Chao and M Tigner (editors), Handbook of Accelerator Physics and Engineering (World Scientific, 1998), Sections 2.7.4, 2.7.5.

[7] E. Courant, BNL Report C-A/AP/292 (2007)

[8] S Y Lee, Accelerator Physics (World Scientific, 1997), eqs. (2.66)-(2.69)

[9] M. Bai, W. MacKay, T. Roser, Phys. Rev. ST AB 8, 099001 (2005)

[10] S Y Lee, Phys. Rev. ST AB 9, 074001 (2006)

[11] A M Kondratenko, M A Kondratenko, Y N Filatov, contribution to Michigan-COSY teleconference $12 / 11 / 07$ 